\title{
Disponibilidade domiciliar de alimentos a partir da nova classificação de alimentos e (in)segurança alimentar
}

\author{
Household availability of food based on the new classification of \\ food and nutritional (in)security
}

Lanay Dias Barbosa (https://orcid.org/0000-0002-9632-0193) ${ }^{1}$

Lohane Karolina Melo Sousa (https://orcid.org/0000-0002-7615-3260) ${ }^{1}$

Eloise Schott (https://orcid.org/0000-0002-3852-1894) ${ }^{1}$

Fabiane Aparecida Canaan Rezende (https://orcid.org/0000-0002-5617-8778) ${ }^{1}$

Andreia Queiroz Ribeiro (https://orcid.org/0000-0001-6546-1252) ${ }^{2}$

Silvia Eloíza Priore (https://orcid.org/0000-0003-0656-1485) ${ }^{2}$

Sylvia do Carmo Castro Franceschini (https://orcid.org/0000-0001-7934-4858) ${ }^{2}$

${ }^{1}$ Departamento de Nutrição, Universidade Federal do

Tocantins. Quadra 109 Norte, Avenida NS 15, Plano Diretor Norte. 77001090 Palmas TO Brasil. lanaydb@gmail.com

${ }^{2}$ Departamento de Nutrição

e Saúde, Universidade

Federal de Viçosa. Viçosa

MG Brasil.

\begin{abstract}
The scope of this research was to characterize the household availability of food based on the classification adopted in the new Brazilian food guide and to analyze its relationship with food (in)security. It involved a population-based, cross-sectional study conducted in 95 households in the State of Tocantins. Food (in)security was evaluated by means of the Brazilian Food Insecurity Scale. The household availability of food in the last 30 days was recorded and, subsequently, food was classified as in natura and/or minimally processed, processed and ultra-processed according to the new Brazilian food guide. The prevalence of food insecurity was $55.79 \%$. It was found that the foods of the in natura and ultra-processed groups contributed the most to the household availability of food. There was a positive and significant relationship between the food security and food availability rates: milk, animal fat, canned food and cheese bread. The prevalence of food insecurity was high in this population group. In households with a higher level of food security, the availability of milk, animal fat, canned goods and cheese bread was significantly higher.

Key words Industrialized food, Nutritional Epidemiology, Food Classifications, Food and nutritional security
\end{abstract}

Resumo O objetivo deste estudo foi caracterizar a disponibilidade domiciliar de alimentos a partir da classificação dos alimentos adotada no novo guia alimentar brasileiro e analisar sua relação com a (in)segurança alimentar. Trata-se de um estudo de base populacional, transversal, realizado em 95 domicílios do Estado do Tocantins. Avaliouse a (in)segurança alimentar, por meio da Escala Brasileira de Insegurança Alimentar. A disponibilidade domiciliar de alimentos nos últimos 30 dias foi registrada e, posteriormente, classificou-se os alimentos em in natura elou minimamente processados, processados e ultraprocessados, conforme o novo guia alimentar brasileiro. A prevalência de insegurança alimentar foi de 55,79\%. Verificou-se que os alimentos dos grupos in natura e ultraprocessados foram os que mais contribuíram para a disponibilidade domiciliar de alimentos. Verificou-se relação positiva e significante do grau de segurança alimentar com a disponibilidade dos alimentos: leite, gordura animal, enlatados e pão de queijo. A prevalência de insegurança alimentar foi elevada nessa população. Nos domicílios com maior grau de segurança alimentar, a disponibilidade de leite, gordura animal, enlatados e pão de queijo foi significantemente maior.

Palavras-chave Alimentos industrializados, Epidemiologia Nutricional, Guias Alimentares, Segurança alimentar e nutricional 


\section{Introdução}

A Lei Orgânica de Segurança Alimentar e Nutricional (Lei no 11.346/2006') define Segurança Alimentar e Nutricional (SAN) como: "o direito de todos ao acesso regular e permanente a alimentos de qualidade, em quantidade suficiente, sem comprometer o acesso a outras necessidades essenciais, tendo como base práticas alimentares promotoras de saúde, que respeitem a diversidade cultural e que sejam social, econômica e ambientalmente sustentáveis".

Dados da última Pesquisa Nacional por Amostra de Domicílios (PNAD) 2013 indicaram que $77,4 \%$ dos domicílios particulares brasileiros encontravam-se em situação de segurança alimentar, sendo este número superior a anos anteriores (65,1\%, em 2004 e 69,8, em 2009). Porém, a insegurança alimentar atingiu $22,6 \%$ da população, representando 14,7 milhões de domicílios particulares, sendo que as regiões Norte e Nordeste apresentaram situações mais preocupantes, com prevalências de $36,1 \%$ e $38,1 \%$, respectivamente ${ }^{2}$.

A SAN engloba diferentes aspectos que podem ser agrupados em dois componentes: o componente alimentar, que é relacionado à produção, disponibilidade, comercialização e acesso a alimentos saudáveis e o componente nutricional que se refere às práticas alimentares, consumo e a utilização biológica dos alimentos em todas as fases do ciclo de vida ${ }^{3}$. Portanto, pode-se dizer que a SAN engloba o acesso a alimentos de qualidade, práticas alimentares saudáveis e direitos humanos ${ }^{4}$. Vale ressaltar ainda que um bom estado nutricional não depende apenas da segurança alimentar, mas também do acesso a outras condições como moradia, saneamento básico, acesso a serviços de saúde e educação ${ }^{5}$.

Não só fatores individuais, como por exemplo necessidades fisiológicas ou nutricionais, mas também contextuais, relacionados ao ambiente, determinam a situação de SAN. Condições ambientais como acesso, disponibilidade e preço dos alimentos interferem nas escolhas alimentares do homem e estão diretamente relacionadas ao maior poder de escolha sobre os alimentos ultraprocessados e, consequentemente, redução do consumo de alimentos in natura e minimamente processados $^{6}$.

No Brasil, a Pesquisa de Orçamentos Familiares $(\mathrm{POF})^{7}$ 2008-2009 realizada pelo Instituto Brasileiro de Geografia e Estatística, destaca a elevação do consumo de carnes e alimentos industrializados (refrigerantes, biscoitos e refeições prontas) e a redução do consumo de leguminosas, raízes e tubérculos, frutas e hortaliças. Esses alimentos ultraprocessados quando comparados com alimentos in natura apresentam maior densidade energética, maior teor de gorduras em geral, de gordura saturadas, de gorduras trans, de sódio e de açúcar livre e menor teor de fibras e proteína $^{8}$. Tal substituição está entre as principais causas de obesidade e Doenças Crônicas Não Transmissíveis (DCNT) ${ }^{9}$.

Recentemente, o debate acerca da relação entre grau de processamento dos alimentos $\mathrm{e}$ doenças crônicas ganhou expressividade, especialmente após o lançamento no Guia Alimentar para a População Brasileira. Segundo Monteiro et al. ${ }^{10}$, o processamento de alimentos é o uso de métodos e técnicas para modificar o alimento fresco em produto alimentício. Existem quatro tipos de categorias de alimentos conforme o processamento utilizado, os in natura que são oriundos diretamente de plantas ou animais sem sofrerem modificações; os minimamente processados que passam por alterações mínimas como cortar, moer, lavar e polir; os processados que envolvem a adição de sal, açúcar, óleos e gorduras e os ultraprocessados passam por várias fases de processamento e possuem ingredientes industriais ${ }^{11}$.

O aumento do consumo e disponibilidade de alimentos processados e ultraprocessados ocorreu, principalmente, após a adoção de novos modos de vida pela sociedade, marcada por uma forma de se alimentar, que prioriza a rapidez, economia e a praticidade ${ }^{12}$. Em razão disso, surgem situações que colocam em risco a segurança alimentar da população devido ao consumo em excesso de alimentos industrializados e baixo consumo de alimentos in natura, contribuindo para a obesidade e deficiência de micronutrientes ${ }^{12}$.

A disponibilidade domiciliar de alimentos auxilia no monitoramento da SAN, pois é um dos indicadores que permite analisar a qualidade da alimentação consumida pelas famílias. Frente ao exposto, o objetivo deste estudo foi caracterizar a disponibilidade domiciliar de alimentos de famílias residentes na zona urbana de quatros municípios do estado do Tocantins a partir da classificação dos alimentos adotada no novo guia alimentar brasileiro e analisar sua relação com a (in)segurança alimentar.

\section{Materiais e métodos}

Os dados deste estudo foram obtidos de uma pesquisa de base populacional, do tipo transver- 
sal, intitulada "Prevalência e fatores associados a (In)segurança alimentar e nutricional no estado do Tocantins", realizada no período de setembro de 2016 a julho de 2017.

Para a referida pesquisa, empregou-se um plano de amostragem complexa por conglomerados, dividido em três estágios. Primeiramente foram sorteados aleatoriamente os municípios, em seguida os setores censitários urbanos de cada município, depois um quarteirão e neste uma esquina que foi utilizada como ponto de partida para a coleta dos dados. A coleta de dados iniciou-se sempre em sentido horário a partir do primeiro domicílio situado na esquina sorteada, e a partir dele, os domicílios subsequentes foram visitados de maneira alternada, sendo um domicílio avaliado, o outro não, e assim sucessivamente.

O cálculo do tamanho da amostra foi realizado por meio do programa OpenEpi ${ }^{\circledR}$, utilizando a equação: $n=\left[\operatorname{EDFF}^{\star} \mathrm{Np}(1-\mathrm{p})\right] /\left[\left(\mathrm{d}^{2} / \mathrm{Z}^{2} 1-/ 2^{\star}(\mathrm{N}\right.\right.$ $\left.-1)+p^{\star}(1-p)\right]$, em que: $N=$ Tamanho da população (para o fator de correção da população finita ou fcp); $\mathrm{p}=$ Frequência \% hipotética do fator do resultado na população; EDFF = Efeito de desenho para inquéritos em grupo e $\mathrm{d}=$ Limites de confiança como \% de 100 (absoluto +/-\%).

Considerou-se para o cálculo: intervalo de confiança (d) de 95\%, erro amostral de 5\% (p) e efeito de desenho igual a 1,5. Foram adicionados $10 \%$ para controle de fatores de confusão. Com isso, foram selecionados 595 domicílios, de 22 municípios, pertencentes às 08 microrregiões do estado, sendo que o número de municípios foi previamente definido a partir do recurso financeiro disponível para a pesquisa, e a quantidade de domicílios avaliados em cada município e microrregião foi proporcionalmente ao tamanho populacional.

O presente estudo refere-se aos dados preliminares de quatro municípios pertencentes à microrregião de Miracema do Tocantins, sendo eles: Itaporã do Tocantins, Miranorte, Abreulândia e Fortaleza do Tabocão, totalizando 95 domicílios avaliados.

Previamente à etapa de coleta de dados, realizou-se um estudo piloto para avaliar o planejamento proposto, o qual possibilitou testar o questionário da pesquisa, treinar os entrevistadores (docentes e acadêmicos do curso de nutrição da Universidade Federal do Tocantins), verificar a logística proposta à amostragem e estimar o tempo médio de coleta por domicílio.

A coleta de dados nos domicílios foi realizada por meio de entrevista estruturada com o chefe de família para levantamento de informações demográficas, socioeconômicas, hábitos de vida, disponibilidade domiciliar de alimentos, situação de Segurança Alimentar e Nutricional (SAN) e estado nutricional avaliado por meio de antropometria. Para este estudo utilizou-se as variáveis: idade, sexo, escolaridade, raça/cor, estado civil, total de pessoas residentes no domicílio, renda per capita, participação em programas assistenciais, situação de SAN e disponibilidade domiciliar de alimentos.

Para a avaliação da situação de Segurança Alimentar da família utilizou-se a Escala Brasileira de Insegurança Alimentar (EBIA), uma escala psicométrica, com 14 perguntas fechadas, referente aos últimos 3 meses, que avalia a percepção da segurança alimentar intrafamiliar em relação ao acesso aos alimentos, além de identificar os grupos vulneráveis à violação do direito humano à alimentação adequada ${ }^{13}$. A situação de Segurança Alimentar da família foi classificada em quatro níveis conforme pontuação a seguir ${ }^{14}:$ a) para domicílios com menores de 18 anos: segurança alimentar: 0 pontos; insegurança alimentar leve: 1 a 5 pontos; insegurança alimentar moderada: 6 a 9 pontos; insegurança alimentar grave: 10 a 14 pontos; b) para domicílios sem menores de 18 anos: segurança alimentar: 0 pontos; insegurança alimentar leve: 1 a 3 pontos; insegurança alimentar moderada: 4 a 5 pontos; insegurança alimentar grave: 6 a 8 pontos.

A disponibilidade dos alimentos foi avaliada a partir de um questionário de disponibilidade de alimentos aplicado pelo pesquisador ao chefe de família, que informou de forma retrospectiva os alimentos e bebidas adquiridos por meio de compra, doação e/ou produção própria nos últimos 30 dias. Os alimentos foram agrupados de acordo com a classificação de alimentos proposta por Monteiro et al. ${ }^{10}$ segundo o tipo de processamento: in natura e/ou minimamente processados, ingredientes culinários e/ou alimentos processados e alimentos ultraprocessados. Posteriormente, os alimentos pertencentes a cada um dos referidos grupos foram agrupados por similaridade conforme composição nutricional predominante (Quadro 1).

Os dados foram tabulados no programa Microsoft Excel $2010^{\varpi}$ e o tratamento estatístico dos dados foi realizado no pacote estatístico Stata versão 13.0. Realizou-se análise descritiva dos dados através de frequência absoluta e relativa para variáveis categóricas ou médias e desvio-padrão para variáveis contínuas. Para verificar a relação entre condição de (in)segurança alimentar e disponibilidade domiciliar de alimentos foi uti- 
Quadro 1. Classificação dos alimentos disponíveis nos domicílios da microrregião de Miracema do Tocantins. Tocantins, Brasil, 2016/2017.

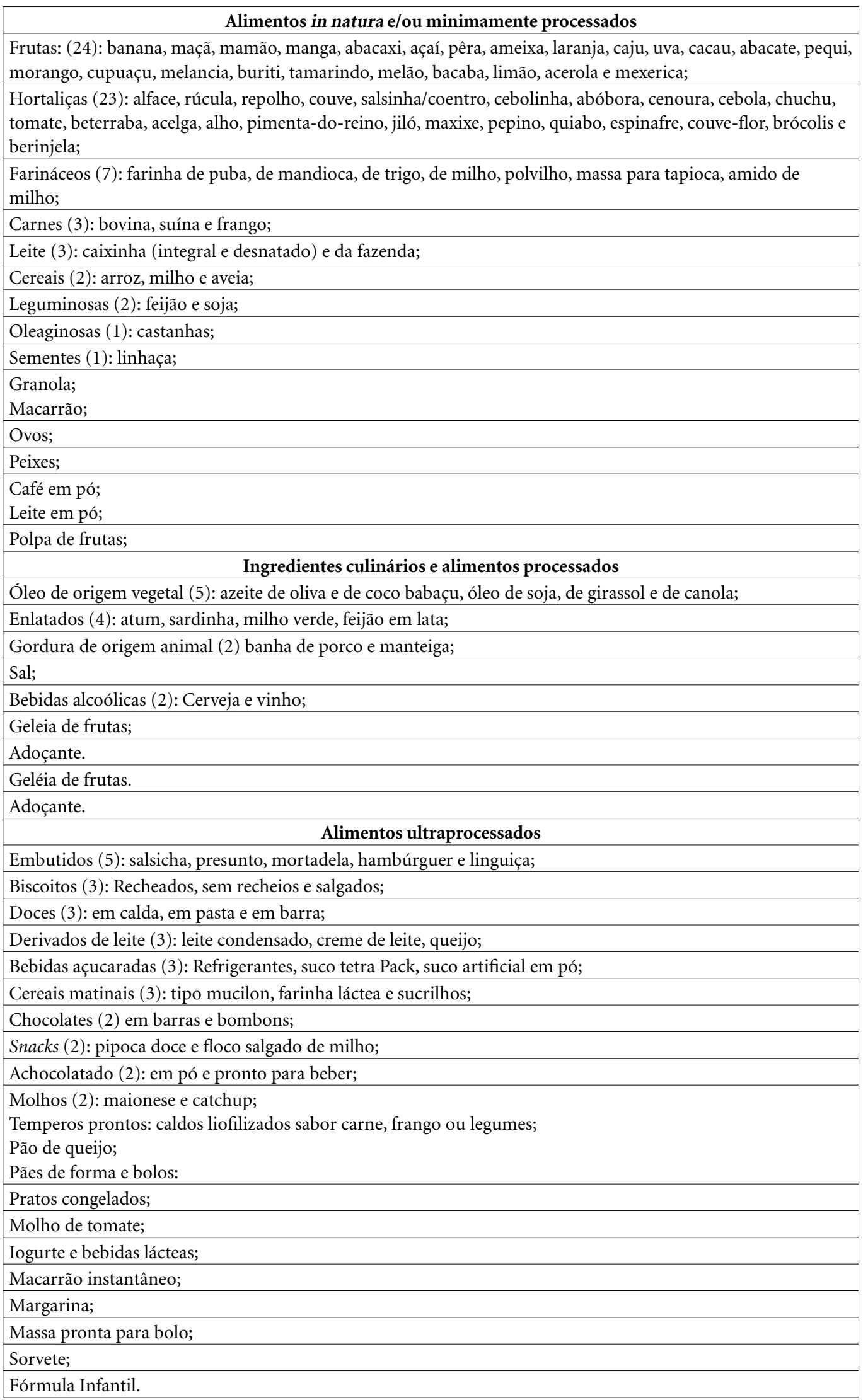


lizado o teste de tendência linear. Para todas as análises adotou-se alfa igual a 5\% como nível de significância estatística. A pesquisa foi aprovada pelo Comitê de Ética em Pesquisa da Universidade Federal de Viçosa e todos os participantes foram esclarecidos sobre o objetivo e procedimentos, sendo incluídos na mesma, após assinarem um Termo de Consentimento Livre e Esclarecido.

\section{Resultados}

A análise dos dados demográficos e socioeconômicos demonstrou que entre os chefes de família $78,95 \%$ eram do sexo feminino, que os mesmos apresentavam em média 49,36 $\pm 18,23$ anos de idade $(\mathrm{n}=95)$ e $9,34 \pm 5,06$ anos de estudo ( $\mathrm{n}=$ 87 ), sendo que $48,42 \%$ se autodeclaram de raça/ cor parda e $43,16 \%$ eram casados. Quanto ao número de moradores por domicílio identificou-se que a média foi de $3,24 \pm 1,68$ pessoas $(\mathrm{n}=95)$. Em relação à renda, cerca de 67,37\% $(n=64)$ dos domicílios apresentavam renda familiar per capita inferior a um salário mínimo, $11,58 \%(\mathrm{n}=11)$ inferior a um quarto do salário mínimo e 21,05\% $(\mathrm{n}=20)$ recebiam algum benefício de programas assistenciais do governo federal. A prevalência de insegurança alimentar nas famílias estudadas foi de 55,79\% ( $\mathrm{n}=53$ ) (Tabela 1).

Quanto à frequência relativa de alimentos segundo o grau de segurança alimentar, verificouse que no grupo in natura e/ou minimamente processados os alimentos mais disponíveis foram aqueles que pertenciam aos grupos dos cereais, hortaliças, carnes, leguminosas, frutas, farináceos, ovos, café em pó, raízes e tubérculos, leite e macarrão. Já no grupo dos ingredientes culinários e alimentos processados, os alimentos mais frequentes foram sal, açúcar e óleo de origem vegetal. E no grupo dos ultraprocessados, molho de tomate, biscoitos, margarina, bebidas açucaradas, pães e bolos e derivados do leite (Tabela 2).

Verificou-se uma relação direta entre grau de insegurança alimentar e menor disponibilidade domiciliar dos seguintes alimentos: leite (teste de tendência linear: $p=0,032$ ), gordura animal (teste de tendência linear: $p=0,012$ ), enlatados (teste de tendência linear: $p=0,018$ ), pão de queijo (teste de tendência linear: $\mathrm{p}=0,016$ ).

\section{Discussão}

No presente estudo verificaram-se altas taxas de insegurança alimentar na microrregião de Mira- cema do Tocantins (55,79\%). Ao comparar com os dados publicados pela $\mathrm{PNAD}^{2}$, verifica-se que o percentual é consideravelmente maior que o do estado do Tocantins (37,6\%), e mais que o dobro da média encontrada no Brasil (22,6\%). Em geral, a Insegurança Alimentar é mais prevalente em domicílios sob vulnerabilidade sociodemográfica e com menor renda per capita ${ }^{15}$, características presentes na população deste estudo.

É relevante contextualizar que os dados desta pesquisa foram coletados no ano de 2016, período no qual análise do Instituto de Pesquisa Econômica Aplicada (IPEA) sobre mercado de trabalho e inflação dos alimentos apontou que houve queda no nível de atividade, com aumento da taxa de desemprego e elevação da inflação, sendo que o Índice Nacional de Preços ao Consumidor Amplo (IPCA) apresentou alta de 6,4\% e o produto interno bruto (PIB) caiu 3,6\% no mesmo ano ${ }^{16}$. O IPCA-15, que mede a inflação da Alimentação no Domicílio, foi de $16,6 \%$, no acumulado dos 12 meses (agosto 2016 a julho de 2017), com destaque negativo para os cereais, frutas, farinhas e massas, leites e derivados ${ }^{17}$.

Nesse contexto entende-se que as políticas públicas que assegurem trabalho, renda e educação assumem grande importância, pois refletem diretamente na Insegurança Alimentar, e consequentemente, no acesso aos alimentos e à alimentação de qualidade.

Este estudo demonstrou um elevado percentual de disponibilidade de alimentos in natura (cereais, hortaliças, carnes, leguminosas, frutas, farináceos, ovos, café em pó, raízes e tubérculos e leite) e, em contrapartida, alimentos processados e ultraprocessados os quais apresentam menor teor de nutrientes, maior densidade energética, gorduras saturadas e trans, açúcar livre e menores teores de fibras e proteínas ${ }^{18,19}$. Alguns estudos mostram a relação consumo de alimentos ultraprocessados com prejuízos à saúde, tais como, síndrome metabólica em adolescentes ${ }^{20}$, obesidade em crianças ${ }^{21} \mathrm{e}$ em todas as idades ${ }^{22}$.

Além disso, segundo estudo de Louzada et al. ${ }^{8}$ o consumo de alimentos ultraprocessados impactou negativamente no perfil nutricional da dieta, pois contribuíram para aumento significativo da densidade energética, do teor relativo de açúcar livre, de gorduras em geral, de gorduras saturadas e de gorduras trans e redução do teor de proteínas, de fibras, de potássio e de sódio.

A relação positiva do grau de segurança alimentar com disponibilidade de alguns alimentos específicos (leite, gordura animal, enlatados e pão de queijo), observado neste estudo, evidencia 
Tabela 1. Características demográficas, socioeconômicas dos chefes de famílias e situação de segurança alimentar e nutricional dos domicílios de quatro municípios pertencentes à microrregião de Miracema do Tocantins. Tocantins, Brasil, setembro de 2016 e julho de 2017.

\begin{tabular}{|c|c|c|}
\hline Variáveis & n (95) & $\%$ \\
\hline \multicolumn{3}{|l|}{ Sexo } \\
\hline Masculino & 20 & 21,05 \\
\hline Feminino & 75 & 78,95 \\
\hline \multicolumn{3}{|l|}{ Cor/Raça } \\
\hline Branco & 24 & 25,26 \\
\hline Preto & 16 & 16,84 \\
\hline Pardo & 46 & 48,42 \\
\hline Amarelo & 7 & 7,37 \\
\hline Indígena & 2 & 2,11 \\
\hline \multicolumn{3}{|l|}{ Estado civil } \\
\hline Solteiro & 18 & 18,95 \\
\hline Casado & 41 & 43,16 \\
\hline Separado & 10 & 10,53 \\
\hline Divorciado & 17 & 17,89 \\
\hline Viúvo & 9 & 9,47 \\
\hline \multicolumn{3}{|l|}{ Grau de escolaridade } \\
\hline Analfabeto & 8 & 8,43 \\
\hline Ensino fundamental incompleto & 32 & 33,68 \\
\hline Fundamental completo & 5 & 5,26 \\
\hline Médio incompleto & 4 & 4,21 \\
\hline Médio completo & 23 & 24,21 \\
\hline Superior incompleto & 7 & 7,37 \\
\hline Superior completo & 16 & 16,84 \\
\hline \multicolumn{3}{|l|}{ Renda per capita } \\
\hline Não informado & 5 & 5,26 \\
\hline Até $1 / 4$ de salário mínimo & 11 & 11,58 \\
\hline De $1 / 4$ a 1/2 salário mínimo & 22 & 23,16 \\
\hline$>1 / 2$ a 1 salário mínimo & 31 & 32,63 \\
\hline$>1$ até 2 salários mínimos & 19 & 20,00 \\
\hline$>2$ salários mínimos & 7 & 7,37 \\
\hline \multicolumn{3}{|l|}{$\begin{array}{l}\text { Beneficiado por programas } \\
\text { assistenciais }\end{array}$} \\
\hline Sim & 20 & 21,05 \\
\hline Não & 75 & 78,95 \\
\hline \multicolumn{3}{|l|}{$\begin{array}{l}\text { Situação de (In)Segurança Alimentar } \\
\text { (EBIA)* }\end{array}$} \\
\hline Segurança Alimentar & 42 & 44,21 \\
\hline Insegurança Alimentar Leve & 27 & 28,42 \\
\hline Insegurança Alimentar Moderada & 18 & 18,95 \\
\hline Insegurança Alimentar Grave & 8 & 8,42 \\
\hline
\end{tabular}

${ }^{\star}$ EBIA: Escala Brasileira de Insegurança Alimentar.

que estar seguro pela EBIA não garantiu o acesso a alimentos de boa qualidade nutricional, visto que houve relação linear positiva entre a melhor condição de SAN e maior disponibilidade de alimentos processados e ultraprocessados, os quais conferem riscos à saúde, principalmente de doenças crônicas ${ }^{23}$. Cabe aqui destacar que o leite é um alimento com boa qualidade nutricional, no entanto, houve predomínio do leite do tipo integral e leite da fazenda, que contribui para maiores quantidades de gorduras saturadas na alimentação. Adicionalmente, é possível que a maior ocorrência de excesso de peso em pessoas vivendo em insegurança alimentar na população brasileira esteja relacionada ao maior consumo destes alimentos ${ }^{24}$.

Apesar dos domicílios em insegurança alimentar terem apresentado menor frequência de disponibilidade de alimentos ultraprocessados, cabe aqui ressaltar que se identificou também menor consumo de alimentos in natura e minimamente processados que também implica em menor qualidade nutricional da alimentação. Martins et al. ${ }^{25}$ avaliaram 200 famílias na zona rural do município de Maranguape, região metropolitana de Fortaleza-CE e verificaram uma tendência significante de consumo decrescente de tubérculos/raízes, o leite, os derivados do leite, as frutas/sucos naturais, as verduras/legumes, as carnes e a gordura, à medida que a Insegurança Alimentar se agravou.

A EBIA é um indicador sensível para identificar famílias em risco de insegurança alimentar, sendo um instrumento relevante para mensurar a escassez ou não do alimento, e isso está atrelado a condição socioeconômica das famílias, porém ela isoladamente não permite verificar a qualidade da dieta ${ }^{26}$. Cabe destacar que deve haver preocupação não somente com a Segurança Alimentar e Nutricional em si, mas também com a necessidade de avaliar a qualidade da dieta, uma vez que neste estudo as pessoas em segurança alimentar identificada pela EBIA tinham maior consumo de alimentos dados como não saudáveis.

Diante disso, é importante reforçar a necessidade de incluir marcadores de consumo que permitam avaliar a qualidade da alimentação, sendo necessários estudos que validem esses marcadores, de acordo com a cultura alimentar local, e os riscos à saúde que se pretende estimar por meio deles.

Além da necessidade de políticas públicas que promovam melhorais na condição socioeconômica das famílias, acredita-se que estratégias que valorizem o consumo de alimentos regionais, que incentivem o plantio e fortaleçam a produção local de alimentos e que promovam a formação/ mudança de hábitos alimentares saudáveis das 
Tabela 2. Frequência relativa de alimentos segundo o grau de segurança alimentar nos domićlios da microrregião de Miracema do Tocantins. Tocantins, Brasil, 2016/2017.

\begin{tabular}{|c|c|c|c|c|c|c|}
\hline \multirow{2}{*}{ Grupos de alimentos } & \multirow{2}{*}{$\mathbf{N}$} & \multirow{2}{*}{$\%$} & \multirow{2}{*}{$\begin{array}{l}\text { Segurança } \\
\text { Alimentar }\end{array}$} & \multicolumn{3}{|c|}{ Insegurança Alimentar } \\
\hline & & & & Leve & Moderada & Grave \\
\hline \multicolumn{7}{|c|}{ Alimentos In natura ou minimamente processados } \\
\hline Cereais & 95 & 100 & 44,21 & 28,42 & 18,95 & 8,42 \\
\hline Hortaliças & 95 & 100 & 44,21 & 28,42 & 18,95 & 8,42 \\
\hline Carnes & 95 & 100 & 44,21 & 28,42 & 18,95 & 8,42 \\
\hline Leguminosas & 93 & 98 & 45,16 & 29,03 & 17,20 & 8,60 \\
\hline Frutas & 92 & 97 & 43,48 & 29,35 & 18,48 & 8,70 \\
\hline Farináceos; & 92 & 97 & 45,65 & 27,17 & 19,57 & 7,61 \\
\hline Ovos; & 89 & 94 & 44,94 & 29,21 & 17,98 & 7,87 \\
\hline Café em pó & 88 & 93 & 43,18 & 28,41 & 20,45 & 7,95 \\
\hline Macarrão & 80 & 84 & 46,25 & 30,00 & 16,25 & 16,25 \\
\hline Leite $^{*}$ & 83 & 87 & 46,99 & 28,92 & 19,28 & 4,82 \\
\hline Peixes & 57 & 60 & 43,86 & 28,07 & 15,79 & 12,28 \\
\hline Polpa de Fruta & 40 & 42 & 47,50 & 30,00 & 17,50 & 5,00 \\
\hline Leite em pó & 36 & 38 & 36,11 & 33,33 & 19,44 & 11,11 \\
\hline Aveia & 21 & 22 & 42,86 & 28,57 & 19,05 & 9,52 \\
\hline Oleaginosas & 5 & 5 & 60,00 & 20,00 & 20,00 & 0,00 \\
\hline Granola & 4 & 4 & 25,00 & 25,00 & 25,00 & 25,00 \\
\hline Sementes & 1 & 1 & 100,00 & 0,00 & 0,00 & 0,00 \\
\hline \multicolumn{7}{|c|}{ Ingredientes culinários e alimentos processados } \\
\hline Sal & 95 & 100 & 44,21 & 28,42 & 18,95 & 8,42 \\
\hline Açúcar & 93 & 98 & 44,09 & 29,03 & 19,35 & 7,53 \\
\hline Óleo de origem vegetal & 89 & 94 & 42,70 & 29,21 & 29,21 & 8,99 \\
\hline Enlatados ${ }^{\star}$ & 48 & 51 & 54,17 & 27,08 & 16,67 & 2,08 \\
\hline Gordura de origem animal ${ }^{\star}$ & 39 & 41 & 56,41 & 28,21 & 15,38 & 0,00 \\
\hline Adoçante & 20 & 21 & 35,00 & 40,00 & 10,00 & 15,00 \\
\hline Bebidas alcoólicas & 21 & 22 & 57,14 & 23,81 & 14,29 & 4,76 \\
\hline Geleia & 9 & 9 & 66,67 & 11,11 & 22,22 & 0,00 \\
\hline \multicolumn{7}{|l|}{ Alimentos Ultraprocessados } \\
\hline Molho de tomate & 82 & 86 & 42,68 & 29,27 & 21,95 & 6,10 \\
\hline Biscoitos & 81 & 85 & 45,68 & 24,69 & 20,99 & 8,64 \\
\hline Margarina & 80 & 84 & 42,50 & 30,00 & 21,25 & 6,25 \\
\hline Bebidas Açucaradas & 75 & 79 & 42,67 & 30,67 & 20,00 & 6,67 \\
\hline Pães e bolos & 73 & 77 & 47,95 & 28,77 & 17,81 & 5,48 \\
\hline Derivados de leite & 72 & 76 & 48,61 & 26,39 & 16,67 & 8,33 \\
\hline Embutidos & 68 & 62 & 44,12 & 27,94 & 19,12 & 8,82 \\
\hline Temperos prontos & 58 & 61 & 44,83 & 27,59 & 18,97 & 8,62 \\
\hline Pão de queijo ${ }^{*}$ & 57 & 60 & 50,88 & 29,82 & 19,30 & 0,00 \\
\hline Achocolatado & 53 & 56 & 45,28 & 26,42 & 22,64 & 5,66 \\
\hline Doces & 43 & 45 & 48,84 & 30,23 & 16,28 & 4,65 \\
\hline Macarrão instantâneo & 41 & 43 & 43,90 & 31,71 & 17,07 & 7,32 \\
\hline Molhos & 37 & 39 & 48,65 & 29,73 & 18,92 & 2,70 \\
\hline Sorvete & 35 & 37 & 57,14 & 14,29 & 22,86 & 5,71 \\
\hline Iogurte e bebidas lácteas & 34 & 36 & 52,94 & 17,65 & 23,53 & 5,88 \\
\hline Massa pronta para bolo & 30 & 32 & 56,67 & 20,00 & 16,67 & 6,67 \\
\hline Snacks & 29 & 31 & 48,28 & 34,48 & 17,24 & 0,00 \\
\hline Cereais matinais & 21 & 22 & 52,38 & 23,81 & 14,29 & 9,52 \\
\hline Chocolates & 18 & 19 & 61,11 & 11,11 & 22,22 & 5,56 \\
\hline Fórmula Infantil & 4 & 4 & 50,00 & 0,00 & 50,00 & 0,00 \\
\hline Pratos congelados & 2 & 2 & 100,00 & 0,00 & 0,00 & 0,00 \\
\hline
\end{tabular}


famílias, podem contribuir para diminuir o consumo de alimentos ultraprocessados e aumentar os de alimentos in natura. Ressalta-se por fim, que este se trata de um estudo preliminar, portanto, não é possível apontar conclusões definitivas até a sua finalização.

\section{Colaboradores}

LD Barbosa e LKM Sousa participaram da coleta, análise, interpretação dos dados e redação do manuscrito. E Schott participou da concepção e delineamento do estudo, coleta de dados, revisão crítica do conteúdo e aprovação final do manuscrito. FAC Rezende participou da concepção e delineamento do estudo, análise, interpretação dos dados, redação do manuscrito, revisão crítica do conteúdo e aprovação final do manuscrito. AQ Ribeiro, SE Priore e SCC Franceschini participaram da concepção e delineamento do estudo.

\section{Agradecimentos}

Os autores do artigo agradecem ao Ministério do Desenvolvimento Social e Agrário pelo financiamento do projeto e ao Conselho Estadual de Segurança Alimentar do Tocantins pelo apoio à pesquisa. 


\section{Referências}

1. Brasil. Lei no 11.346 , de 15 de setembro de 2006. Cria o Sistema Nacional de Segurança Alimentar e Nutricional - SISAN, com vistas em assegurar o direito humano à alimentação adequada e dá outras providencias. Diário Oficial da União 2006; 18 set.

2. Instituto Brasileiro de Geografia e Estatística (IBGE). Pesquisa Nacional por Amostra de Domicílios: segurança alimentar 2013. Rio de Janeiro: IBGE; 2014.

3. Pedraza DF, Sales MC. (In)segurança alimentar e nutricional: desenvolvimento de um indicador do problema e experimentação em famílias da Paraíba, Brasil. Interações 2014; 15(1):79-88.

4. Kepple AW, Segall-Corrêa AM. Conceituando e medindo Segurança Alimentar e Nutricional. Cien Saude Colet 2011; 16(1):187-199.

5. Hoffmann R. Pobreza, insegurança alimentar e desnutrição no Brasil. Estud Av 1995; 9(24):159-172.

6. Claro RM, Maia EG, Costa BVL, Dani DP. Preço dos alimentos no Brasil: prefira preparações culinárias a alimentos ultraprocessados. Cad Saúde Pública 2016; 32(8):e00104715.

7. Instituto Brasileiro de Geografia e Estatística (IBGE). Pesquisa de Orçamentos Familiares 2008-2009: avaliação nutricional da disponibilidade de alimentos no Brasil. Rio de Janeiro: IBGE; 2010.

8. Louzada MLC, Martins APB, Canella DS, Baraldi LG, Levy RB, Claro RM, Moubarac JC, Cannon G, Monteiro CA. Alimentos ultraprocessados e perfil nutricional da dieta no Brasil. Rev Saúde Pública 2015; 49:38.

9. Claro FM, Santos MAS, Oliveira TP, Pereira CA, Szwarcwald CL, Malta DC. Consumo de alimentos não saudáveis relacionados a doenças crônicas não transmissíveis no Brasil: Pesquisa Nacional de Saúde, 2013. Epidemiol Serv Saúde 2015; 24(2):257-265.

10. Monteiro CA, Cannon G, Levy RB, Moubarac JC, Jaime P, Martins AP, Canella D, Louzada ML, Parra D; with Ricardo C, Calixto G, Machado P, Martins C, Martinez E, Baraldi L, Garzillo J, Sattamini I. NOVA. A estrela brilha. [Classificação dos alimentos. Saúde Pública]. World Nutr 2016; 7(1-3):28-38.

11. Brasil. Ministério da Saúde (MS). Guia alimentar para a população brasileira. 2a ed. Brasília: MS; 2014.

12. Leão ALM, Santos LC. Consumo de micronutrientes e excesso de peso: existe relação? Rev Bras Epidemiol 2012; 15(1):85-95

13. Perez-Escamilla R, Segall-Corrêa AM, Maranha LK, Sampaio MFA, Marín-León L, Panigassi G. An adapted version of the U.S. Department of Agriculture Food Insecurity Module is a valid tool for assessing household food insecurity in Campinas, Brazil. J Nutr 2004; 134(8):1923-1928.

14. Brasil. Ministério do Desenvolvimento Social e Combate à Fome (MDS). Secretaria de Avaliação e Gestão da Informação (SAGI). Escala Brasileira de Insegurança Alimentar. EBIA: análise psicométrica de uma dimensão da Segurança Alimentar e Nutricional. Estudo técnico. Brasília; SAGI; 2014.
15. Ribeiro CSG, Pilla MCBA. Segurança alimentar e nutricional: interfaces e diminuição de desigualdades sociais. Demetra 2014; 9(1):41-52.

16. Instituto de Pesquisa Econômica Aplicada (IPEA). Boletim Mercado de Trabalho - Conjuntura e Análise no 62. Brasília: IPEA; 2017.

17. Instituto de Pesquisa Econômica Aplicada (IPEA). Carta de Conjuntura $n^{\circ}$ 36. Rio de Janeiro: IPEA; 2017.

18. Monteiro CA, Levy RB, Claro RM, Castro IRR, Cannon G. Increasing consumption of ultra-processed foods and likely impact on human health: evidence from Brazil. Public Health Nutr 2010; 14(1):5-13.

19. Moubarac JC, Martins AP, Claro RM, Levy RB, Canhão G, Monteiro CA. Consumption of ultra-processed foods and likely impact on human health. Evidence from Canada. Public Health Nutr 2012; 16(12):2240-2248.

20. Tavares LF, Fonseca SC, Rosa MLG, Yokoo EM. Relationship between ultra-processed foods and metabolic syndrome in adolescents from a Brazilian Family Doctor Program. Public Health Nutr 2012; 15(1):8287.

21. Sparrenberger K, Friedrich RR, Schiffne MD, Schuch I, Wagne MB. Consumo de alimentos ultraprocessados entre crianças de uma Unidade Básica de Saúde. $J$ Pediatr 2015; 91:535-542.

22. Canella DS, Levy RB, Martins AP, Claro RM, Moubarac JC, Baraldi LG, Cannon G, Monteiro CA. Ultra-processed food products and obesity in Brazilian households (2008-2009). PLoS One 2014; 9(3):e92752.

23. World Health Organization (WHO). Diet, nutrition and the prevention of chronic diseases: Report of a Joint WHO/FAO Expert Consultation. Geneva: WHO; 2003.

24. Schlüssel MM, Silva AAM, Pérez-Escamilla R, Kac G. Household food insecurity and excess weight/obesity among Brazilian women and children: a life-course approach. Cad Saúde Pública 2013; 29(2):219-226.

25. Martins MC, Aires JS, Dantas KB, Sabino LMM, Alves MDS, Ximenes LB. Consumo alimentar de famílias de pré-escolares em situação de (in)segurança alimentar. Cien Enfermeria XXI 2015; 3:63-71.

26. Azevedo E, Ribas MTGO. Estamos seguros? Reflexões sobre indicadores de avaliação da segurança alimentar e nutricional. Rev Nutr 2016; 29(2):241-251.
Artigo apresentado em 04/07/2018

Aprovado em 15/11/2018

Versão final apresentada em 17/11/2018 
